\title{
Performance evaluation and chemical recyclability of a polyethylene/poly(3- hydroxybutyrate-co-3-hydroxyvalerate) blend for sustainable packaging
}

\begin{abstract}
In this study, the use of PE and poly(3-hydroxybutyrate-co-3-hydroxyvalerate) (PHBV) blend films for packaging was investigated by evaluating the performance of the plastic blend and its recyclability. It was found that mechanical properties (tensile strength, elongation at break and Young's modulus) of PE/PHBV blend films containing up to $30 \%$ PHBV were comparable to those of commercial packaging plastics. Moreover, the addition of PHBV reduced the oxygen transmission rate (OTR) by $19 \ddot{1} 25 \%$ and increased the water vapor transmission rate (WVTR) compared to those of neat PE. These properties are greatly applicable for food packaging. Meanwhile, pyrolysis of PE/PHBV films at $310{ }^{\circ} \mathrm{C}$ revealed that the PHBV fraction was completely degraded into its volatile monomers and oligomers indicating that the two polymers can be separated from each other. Gas chromatography-mass spectrometry (GC-MS) and proton nuclear magnetic resonance (1H-NMR) analyses of the PHBV pyrolyzates confirmed that there was no contamination from the PE fraction, suggested the possibility of PHBV chemical recycling. Separated PE on the other hand can be further heated for either chemical or mechanical recycling. The ability to recycle PE and PHBV as proposed herewith promotes recycling and cascade utilization of polymeric materials which may contribute positively to the sustainable packaging.
\end{abstract}

Keyword: Poly(3-hydroxybutyrate-co-3-hydroxyvalerate); Plastic blend; Recyclability; Sustainable packaging 